\title{
Enredando bosques y comunidades: territorialización de REDD+ en el ejido Felipe Carrillo Puerto, México*
}

Cómo citar este artículo: Tobasura Morales, D., Gurri, F., Blanco Wells, G., y Schmook, B. (20r8). Enredando bosques y comunidades: territorialización de REDD+ en el ejido Felipe Carrillo Puerto, México. Cuadernos de Desarrollo Rural, I5 (8I), I-I8. https://doi.org/I0.III44/Javeriana.cdri5-8I.ebct

David Tobasura Morales

El Colegio de la Frontera Sur, México

ORCID: http://orcid.org/0000-0002-8123-6175

Francisco Gurri

El Colegio de la Frontera Sur, México

fgurri@ecosur.mx

ORCID: http://orcid.org/0000-0003-3753-237x

Gustavo Blanco Wells

Universidad Austral de Chile, Chile

ORCID: http://orcid.org/0000-0002-4980-3424

Birgit Schmook

El Colegio de la Frontera Sur, México

ORCID: http://orcid.org/0000-000I-5775-0310

DOI: https://doi.org/10.III44/Javeriana.cdri5-8r.ebct

Redalyc: http://www.redalyc.org/articulo.oa?id=I1757604004

Recibido: 2I Mayo 20I7 I Publicación: I8 Junio 2018

\section{Resumen:}

Analizamos los efectos discursivos y relacionales del proyecto piloto Reducción de Emisiones por Deforestación y Degradación (REDD+) en unidades domésticas con y sin derechos de acceso a la tierra en el ejido Felipe Carrillo Puerto, Quintana Roo. A partir de encuestas y entrevistas semiestructuradas, identificamos dispositivos que favorecen la adopción de prácticas de conservación por parte de los ejidatarios propietarios de la tierra, pero que restringen el manejo forestal tradicional. Estas dinámicas generan cambios en las estrategias de subsistencia de las unidades domésticas y limitan la participación y distribución de beneficios a mujeres, jóvenes y avecindados sin propiedad de la tierra.

Palabras clave: REDD+, políticas ambientales, estrategias adaptativas, acceso y uso a los bosques. 


\title{
Entangling the Forests and the Communities: Territorialization of REDD+ in the Common Land Felipe Carrillo Puerto, Mexico
}

\begin{abstract}
We analyzed the discursive and relational effects of the pilot project 'Reduction of Emissions due to Deforestation and Forest Degradation' (REDD+) in domestic units both entitled and not entitled to land access in the common land Felipe Carrillo Puerto, Quintana Roo. Based on semi-structured surveys and interviews, we identified those mechanisms favoring the adoption of preservation practices by the common land owner-residents while restricting the traditional forest management. These dynamics bring about changes in the subsistence strategies of the domestic units and limit the participation and benefit distribution to women, the youth, and settlers who do not own the land.
\end{abstract}

Keywords: REDD+, environmental policies, adaptative strategies, use and access to forests

\section{Introducción}

La estrategia de Reducción de Emisiones por Deforestación y Degradación (REDD+), impulsada desde 2005 en la XI Conferencia sobre Cambio Climático (COPII), es una iniciativa internacional para la mitigación de las emisiones de gases de efecto invernadero, particularmente del $25 \%$ atribuido a los sectores agricultura, silvicultura y otros usos del suelo (Intergovernmental Panel on Climate Change [IPCC], 20I4). REDD+, como el más reciente marco político global de cambio climático y gobernanza forestal, impulsa desde mecanismos de mercado (Pistorius et al., 20I2) prácticas agrícolas y silvícolas sostenibles e iniciativas de manejo y conservación forestal comunitarias (Cronkleton, Bray y Medina, 20II). También promueve la creación de sistemas multinivel (global-nacional-local) de gobernanza y de medición (Gupta, Lövbrand, Turnhout y Vijge, 20r2) y comercialización de la captura de carbono en bosques tropicales del sur global (Holmgren, 2013).

REDD+ plantea una serie de compromisos sociales a través de salvaguardas y cobeneficios de reducción de la pobreza, seguridad en la tenencia de la tierra y protección de los derechos de pueblos indígenas y campesinos dependientes de los bosques (Larson, 20II). Sin embargo, en la territorialización de proyectos enmarcados en la estrategia, se han evidenciado desafíos como: cambios en las condiciones legales y los acuerdos locales de tenencia y uso de la tierra (Awono, Somorin, Eba’a Atyi y Levang, 20I4; Sunderlin et al., 20I4), distribución inequitativa de beneficios y control de élites locales (Pasgaard, 2015; Poudyal et al., 2016) y exclusión de las mujeres en la toma de decisiones (Larson et al., 2015), entre otros.

Frente a estos retos sociales y en medio de la incierta consolidación política de REDD+ a nivel global y nacional-en México la Estrategia Nacional de REDD+ fue presentada en 2017 (Comisión Nacional Forestal [Conafor], 2017)-, los estudios sobre sus avances y efectos se pueden dividir en dos grandes campos de investigación. El primero, más afianzado, se enfoca en los agentes e instituciones gobiernos, entidades multilaterales, ONG, el sector financiero e industrial- y en los mecanismos y espacios de construcción discursiva de REDD+ como marco de gobernanza de los bosques y de la población que depende de ellos (Corbera y Schroeder, 20II; Holmgren, 20I3; Newton, Miller, Byenkya, y Agrawal, 20I6). En el segundo campo, se indaga cómo el marco de gobernanza de REDD+ se traduce o materializa en iniciativas subnacionales y cómo se integran, legitiman e inciden en contextos diferentes a los de la negociación política, como los espacios comunitarios (Benjaminsen, 2017; Gallemore, Gregorio, Brockhaus, Fatorelli y Muharrom, 2017; Pasgaard, 2015). 
El presente artículo se inserta en este último campo a través de una investigación sobre los efectos de la territorialización discursiva de REDD + y a partir de su traducción por parte de agentes intermediarios locales y de su reproducción al interior de un ejido mexicano. Los ejidos, junto con las comunidades agrarias, son las formas jurídicas colectivas de tenencia de la tierra y de aprovechamiento de los bienes comunes en México (Morett-Sánchez y Cosío, 2017). Bajo estas dos modalidades de propiedad se encuentran cerca de 105 millones de hectáreas forestales de las I42 millones del país (Merino, Rodríguez, Ortiz y García, 2008), aprovechadas a través de diversos mecanismos y prácticas de manejo forestal comunitario (Bray, Merino y Barry, 2007).

El ejido Felipe Carrillo Puerto (FCP), Quintana Roo, seleccionado como estudio de caso, refleja este contexto particular de propiedad colectiva de los bosques. Abarca 47.223 ha, de las cuales más del $75 \%$ están cubiertas por selva tropical en diferentes estados de conservación. Estas áreas están destinadas en su mayoría al aprovechamiento forestal y agrícola, con una porción de su superficie destinada a la protección (García-Frapolli, 2015).

Una parte de la superficie de conservación es la reserva comunitaria Much Kanan K’aax (MKK), gestionada por 227 propietarios o ejidatarios con asesoría gubernamental y de una ONG local. En esta área se ha desarrollado una iniciativa nacional de medición, reporte y verificación de captura de carbono (Sánchez-Santos et al., 2015) y se ha emprendido un proceso de certificación internacional para la venta de bonos de carbono (Servicios Ecosistémicos de la Selva Maya [SESM], 20II), por lo que es reconocida a nivel nacional como un proyecto piloto REDD+.

En este contexto, el artículo analiza el proceso de traducción y reproducción discursiva de REDD+ al interior del ejido y su relación con los cambios en los acuerdos locales de tenencia, acceso y uso de la selva a partir de la implementación del proyecto piloto en MKK. El artículo se estructura de la siguiente forma: una primera sección sobre el abordaje conceptual de la territorialización y su aplicación al campo de los discursos y estrategias adaptativas del cambio climático, seguida por dos secciones en las que se describen el lugar de estudio y las herramientas metodológicas utilizadas. Posteriormente, en la sección de resultados, se identifican las dinámicas de uso y acceso a la selva por parte de las unidades domésticas (UD), los dispositivos de reproducción y materialización del discurso de REDD+ y el papel de los agentes intermediarios en el proceso de territorialización. En la discusión se analizan los intereses y el proceso de traducción discursiva y, posteriormente, se abordan los efectos de la territorialización de REED+ en las dinámicas de aprovechamiento y las relaciones entre las UD y la selva. Finalmente, en las conclusiones, se abre el debate sobre las formas de territorialización de REDD+y sus consecuencias locales en espacios rurales.

\section{Territorializar la mitigación del cambio climático: discursos y estrategias adaptativas}

La noción de territorialización es una herramienta conceptual y metodológica que, entre otras cosas, permite dar cuenta del proceso de apropiación social desde los territorios de objetos, ideas, políticas y discursos producidos desde espacios considerados como extraterritoriales (Blanco y Fuenzalida, 2013). El concepto, adaptado de la filosofía deleuziana por Manuel DeLanda bajo el rótulo de teoría del ensamblaje, ha adquirido cierta relevancia por su flexibilidad para describir procesos emergentes de transformación no dicotómicos, en los que se destaca la relacionalidad e interdependencia entre varios agentes, así como la indeterminación en la configuración de nuevas entidades sociales (Anderson y McFarlane, 20II; Blanco, Arce y Fisher, 2015; Woods, 2015). 
En los procesos de territorialización, según DeLanda (2006), se redefinen identidades, fronteras, materialidades e interacciones espaciotemporales. En la teoría del ensamblaje, la espacialidad se establece relacionalmente, y por lo tanto, categorías sociológicamente importantes en la producción del espacio, como el ejercicio del poder, no se encuentran predeterminadas por la posición presente o histórica de los agentes humanos -y no humanos-, sino por los efectos visibles de transformaciones que devienen en nuevas relaciones espaciales fruto de acuerdos, alianzas y resistencias que deben ser empíricamente elucidadas (Anderson y McFarlane, 20rI). La relevancia de un marco basado en el ensamblaje es la posibilidad de analizar las relaciones socioespaciales no desde una perspectiva de jerarquización preestablecida, sino desde la interconexión y transitoriedad, en este caso entre espacios globales, nacionales y locales que interactúan a partir del proyecto piloto REDD+, y de esta forma identificar sus efectos en nuevas prácticas territoriales.

Nuestro punto de partida fue el discurso, entendido como una práctica social regulada y estructurada de uso y difusión de signos o símbolos y de generación de dispositivos y efectos de poder (Keller, 20II). Desde esta definición, el discurso y su estudio no solo abarcan las declaraciones textuales de interpretación o producción de problemas, clasificaciones y subjetividades (Holmgren, 2013), sino también la generación de dispositivos que son utilizados por distintos agentes (institucionales, comunitarios, de mercado...) para reproducirlos, traducirlos y convertirlos en normas, proyectos, instituciones y códigos de conducta (Keller, 20II, 2012).

De esta forma, a partir del análisis del discurso fue posible identificar, en primer lugar, el entramado de agentes e instituciones que inciden en la implementación del proyecto piloto REDD+ y, en segundo lugar, establecer el proceso simbólico (reproducción y traducción) y material (creación de dispositivos) de territorialización de la iniciativa y sus efectos dentro de las relaciones de tenencia, uso y acceso de la selva por parte de los habitantes del ejido.

Para identificar la incidencia de los agentes en los procesos simbólicos y materiales de territorialización, se analizó el papel de los intermediarios locales por su función de mediación entre las instituciones y la sociedad local. Su importancia se debe a que operan entre las interfaces de los distintos niveles espaciales y tienen un importante rol de negociación y representación (Pasgaard, 2015). Para analizar los efectos en la relaciones socioespaciales, partimos de las UD, ya que, gracias a su heterogeneidad en territorios rurales, permiten representar las dinámicas económicas y sociales que afectan la vida y organización comunitaria (Harris, 1986; Wilk, 1994).

La utilización de las UD busca superar los supuestos comunitarios de homogeneidad, consenso y armonía (Agrawal y Gibson, 1999), así como resaltar las dimensiones de tenencia y de género. La primera dimensión se tiene en cuenta por su relevancia en otros estudios sobre gestión territorial en FCP a raíz de la división entre propietarios (ejidatarios) y no propietarios (avecindados) (García-Frapolli, 2015; Méndez-López, García-Frapolli, Ruiz-Mallén, Porter-Bolland y Reyes-García, 2015; Moure-Peña, 2013). Asimismo, la dimensión de género permite visibilizar divisiones socioespaciales de uso y acceso a los bosques entre hombres y mujeres, así como transformaciones en estas dinámicas por cuenta de iniciativas de desarrollo que inciden directamente sobre la propiedad y la importancia de los bienes comunes forestales (Rocheleau y Edmunds, 1997).

Los efectos sobre las relaciones socioespaciales y las dinámicas de uso y acceso a la selva por parte de las UD fueron identificados a partir del enfoque de estrategias adaptativas.Estas son entendidas como marcos de acción que despliegan las UD para responder a sus necesidades e interactuar con condiciones ecosistémicas y marcos regulatorios propios o externos (Gurri, 2010). Su estudio se da a través de los llamados ejes adaptativos: el eje cultural, que revela la organización de las unidades de acuerdo con la apropiación de normas y reglas internas 
o formuladas por agentes externos (Wilk, I994), en este caso del proyecto piloto REDD+, y el eje productivo o estructural, relacionado con las actividades de producción, reproducción y mantenimiento de los miembros de la unidad (Gurri, 2010; McNetting, Wilk y Arnould, 1985), a partir del cual se identificaron las dinámicas de uso, acceso y dependencia a la selva.

En suma, a partir del concepto de territorialización proveniente de la teoría del ensamblaje, se hará un análisis sobre cómo distintos agentes locales, particularmente intermediarios o promotores y miembros de UD del ejido FCP, traducen, reproducen y materializan discursivamente una estrategia que conecta distintos niveles espaciales, como lo es REDD+. Adicionalmente, se estudiará cómo estos agentes generan nuevas formas de relación socioespacial a partir de cambios en sus estrategias adaptativas.

\section{Lugar de estudio: Felipe Carrillo Puerto y la reserva Much Kanan K’aax}

Este territorio, ubicado al centro del estado de Quintana Roo, fue declarado como ejido forestal en 1947 (U’yo'olché A. C., 2005). En él habitan actualmente 288 ejidatarios y sus familias, además de los avecindados que son hijos mayores de ejidatarios o residentes que acceden a la tierra por compra o arriendo (Congreso de los Estados Unidos Mexicanos, 1992). La población del ejido, mayoritariamente maya peninsular, desde su origen ha usado la selva a través de la explotación del chicle extraído del árbol del chicozapote (Manilkara zapota); la concesión a empresas paraestatales para la explotación de cedro (Cedrela odorata L.) y caoba (Swietenia macrophylla), entre 1950-1980; la posterior participación en la asociación de ejidos forestales, hasta 1994, y actualmente por medio de la autogestión forestal de especies comerciales en 25.000 ha (Ellis y Porter-Bolland, 2008; U'yo’olché A. C., 2005).

Además del aprovechamiento maderable, en la selva, gran parte de la población se dedica a la agricultura de subsistencia en milpas -sistema de cultivo desarrollado alrededor del maíz (Granados, Florencia, Ríos y Murcia, 1999)-, principalmente bajo la técnica de roza, tumba y quema (RTQ), y a la recolección de leña y de materiales para la construcción de viviendas (SESM, 20II). En materia de biodiversidad, se encuentran los ecosistemas de selva mediana subcaducifolia, mediana subperennifolia y baja inundable, que coexisten con parches de vegetación secundaria, milpas y pastizales (figura I). Asimismo, el ejido hace parte del corredor biológico entre la Reserva de la Biosfera (RB) de Sian Ka’an y la RB de Calakmul, reconocido por instituciones como la WWF y la Fundación Carlos Slim como “uno de los sumideros de carbono más importantes de México”.

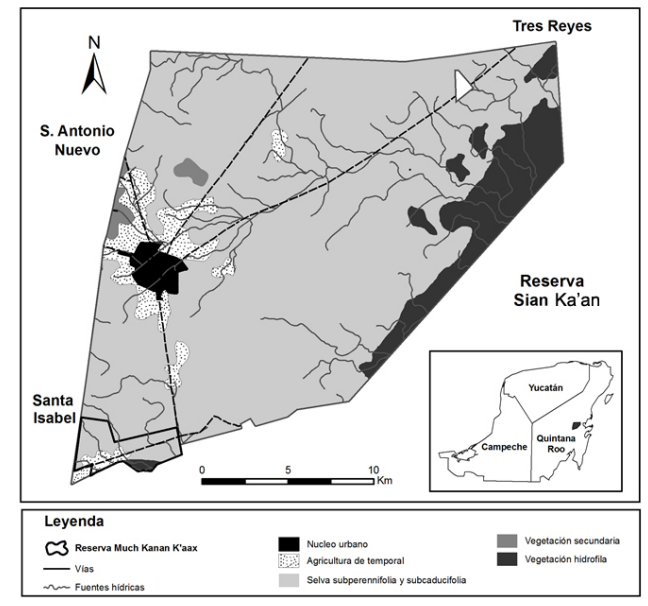

FIGURA i.

ÁREA DE ESTUDIO, EJIDO FELIPE CARRILLO PUERTO, QUINTANA ROO

FUENTE: ELABORACIÓN PROPIA CON BASE EN INFORMACIÓN DEL INSTITUTO NACIONAL DE ESTADÍSTICA Y GEOGRAFÍA (INEGI) 
En el año 2005, los ejidatarios, junto con la ONG U'yo’olché A. C., emprendieron un estudio de ordenamiento territorial con financiación del Banco Mundial y la Conafor. En este proceso se designó la creación de dos áreas de conservación ejidal, la primera, de Io.024 ha, en el límite oriental con la RB Sian Ka’an (U’yo’olché A. C., 2005), y la segunda, nombrada como Much Kanan K’áax (MKK) (“juntos cuidemos la selva”), en el borde suroccidental, con un área de 1230 ha de vegetación madura y secundaria con parches de cultivos. En esta zona se desarrolla un proyecto de captura de carbono gestionado por los ejidatarios y U'yo’olché A. C. (Sánchez-Santos et al., 2015).

El proyecto se ha materializado con dos iniciativas ligadas directamente a REDD+: el monitoreo intensivo de carbono y el proceso de certificación para venta de bonos de carbono. El proyecto Sitios de Monitoreo Intensivo de Carbono, finalizado en 2016 (Sánchez-Santos et al., 2015), cubrió 900 ha de la reserva y formó parte del programa “Fortalecimiento REDD+ y cooperación Sur-Sur”, financiado por el Gobierno de Noruega con asesoría del Programa de las Naciones Unidad para el Desarrollo (PNUD).

Mientras tanto, la iniciativa de certificación es promovida por el ejido y U’yo’olché A. C., con apoyo de la ONG The Nature Conservancy (TNC). Esta inició con un monitoreo de servicios ambientales en 2007, pero solo hasta 20 I fue reconocido como proyecto piloto ante la fundación escocesa Plan Vivo (SESM, 20II), que ofrece servicios de certificación a proyectos comunitarios de pago por servicios ambientales (PSA).

Los objetivos del proyecto piloto son, según sus promotores, promover y proteger la recuperación de la selva tropical por su importancia cultural y ecológica y generar ingresos adicionales a largo plazo para mejorar el manejo forestal comunitario (SESM, 20II). Pese a que el proyecto aún no ha sido certificado, en la reserva ya se han implementado distintas iniciativas, como el ecoturismo, monitoreos comunitarios de la biodiversidad, PSA (García-Frapolli, 2015), reforestación y la disposición de normas de acceso y uso de la selva.

\section{Métodos}

La investigación se estructuró en dos momentos o ejes analíticos: el primero fue la caracterización de las dinámicas de uso y acceso a la selva por parte de las UD, y el segundo eje constó de un análisis discursivo que incluyó la identificación de los agentes intermediarios y, posteriormente, la indagación de su papel en el proceso de reproducción, traducción y materialización discursiva de REDD+ en el ejido.

Entre los meses de enero y junio de 20I6, se realizó una encuesta a 8I personas sobre el uso y acceso a los bienes comunes forestales, y, posteriormente, 23 entrevistas semiestructuradas para identificar la territorialización discursiva de REDD+ e indagar sus efectos en el territorio. Para la implementación de la encuesta, se utilizó un muestreo estratificado únicamente de aquellas UD que han interactuado con las diferentes iniciativas del proyecto piloto en MKK. A partir de una técnica de cuotas (Pimienta-Lastra, 2000), se determinó la base muestral de acuerdo con la condición de tenencia de la tierra del jefe o la jefa de la UD, su posición dentro de esta y su género.

Las mujeres y hombres encuestados pertenecen a $28 \mathrm{UD}$ (tabla I), que fueron seleccionadas de una lista de 40 unidades identificadas por autoridades ejidales y por U’yo’olché A. C. Se utilizó un listado predeterminado de actividades agrícolas y forestales a partir del cual se diseñó un cuestionario sobre las prácticas domésticas y productivas que realizan las personas, la condición de tenencia del terreno y el tipo de vegetación en donde efectúan estas prácticas, con el interés de establecer divisiones espaciales de acuerdo con el género y la tenencia, así como la importancia que tienen para la subsistencia de sus UD. A través del modelo de regresión logística, se estimaron las razones de probabilidad por medio de los coeficientes de regresión, y con la prueba estadística de Wald, se determinó la significancia de las variables dentro del modelo. De esta forma, identificamos asociaciones entre las actividades forestales y el género, la posición y el tipo de tenencia de las UD. 
TABLA 1.

Personas encuestadas según características del muestreo

\begin{tabular}{lllllll}
\hline $\begin{array}{l}\text { Tipo de } \\
\text { tenencia }\end{array}$ & $\begin{array}{l}\text { UD } \\
\text { encuestadas }\end{array}$ & $\begin{array}{l}\text { Personas } \\
\text { encuestadas }\end{array}$ & Género & & \multicolumn{2}{c}{ Posición en la UD } \\
\hline & & & H & M & Jefes & Jóvenes \\
\cline { 3 - 6 } Ejidatario & 22 & 63 & 33 & 30 & 42 & 21 \\
Avecindado & 6 & 18 & 10 & 8 & 12 & 6 \\
\hline
\end{tabular}

Fuente: elaboración propia

Las entrevistas semiestructuradas fueron realizadas a 19 personas -6 mujeres y 13 hombres - ejidatarias y avecindadas que fueron seleccionadas de acuerdo con su participación u oposición a iniciativas ligadas al proyecto piloto REDD+.

Adicionalmente, se entrevistaron 4 representantes de las instituciones que han acompañado la implementación del proyecto en el territorio: la Conafor, por medio de su enlace REDD+, y U'yo’olché A. C.

La realización y sistematización de entrevistas y documentos técnicos siguieron los preceptos de la teoría fundamentada planteados por Keller (20II) en su propuesta de análisis del discurso de la sociología del conocimiento. Este enfoque propone estudiar el discurso a través del orden sociohistórico y espacial en que se produce y del análisis a profundidad de su contenido. Para esta última parte, se utilizaron varios componentes de análisis: los esquemas interpretativos o términos centrales que circulan en el discurso y la estructura del fenómeno o la construcción del problema a partir de declaraciones, posiciones subjetivas, juicios de valor y la generación de prácticas-modelo (Keller, 20II). Adicionalmente, se identificaron los dispositivos (artefactos, planes y sistemas de monitoreo, entre otros) ligados al discurso para establecer su materialización en el territorio. Este enfoque ha sido utilizado por Holmgren (2013) para analizar el campo discursivo global de REDD+.

\section{Resultados}

Esta sección tiene el siguiente esquema narrativo: una primera parte de caracterización del uso y acceso a la selva por parte de las UD del ejido de acuerdo con las categorías de género, tenencia y posición en la unidad; un recuento del proceso de territorialización del proyecto piloto a partir de sus dispositivos normativos, y, posteriormente, se analiza el papel de los intermediarios locales en la interacción entre agentes extraterritoriales y la comunidad, así como su papel en el proceso de traducción y reproducción discursiva del proyecto.

\section{Uso, acceso y dependencia de la selva}

Las actividades productivas y domésticas de subsistencia que realizan los miembros de las UD ejidatarias y, en mayor medida, las avecindadas están relacionadas con la selva. En cuanto al uso de la selva, de acuerdo con el género (tabla 2), se estableció que la cacería, el aprovechamiento maderable, la apicultura y el cultivo de la milpa, así como el apoyo en actividades investigativas y de ecoturismo, son actividades realizadas principalmente por los hombres, mientras que la recolección de leña tiende a ser realizada en mayor medida por las mujeres. 
TABLA 2

ACTIVIDADES EN LA SELVA POR GÉNERO Y TIPO DE TENENCIA

\begin{tabular}{|c|c|c|c|c|c|c|}
\hline \multirow[b]{2}{*}{ Actividades } & \multicolumn{3}{|l|}{ Género } & \multicolumn{3}{|c|}{ Tenencia de la tierra } \\
\hline & $\begin{array}{l}\text { Porcentaje } \\
\text { de } \\
\text { hombres }\end{array}$ & $\begin{array}{l}\text { Porcentaje } \\
\text { de } \\
\text { mujeres }\end{array}$ & Wald & $\begin{array}{l}\text { Porcentaje } \\
\text { de UD } \\
\text { ejidatarias }\end{array}$ & $\begin{array}{l}\text { Porcentaje } \\
\text { de UD } \\
\text { avecindadas }\end{array}$ & Wald \\
\hline Milpa & 58,1 & 18,4 & $9,7^{*}$ & 39,7 & 38,9 & 1,05 \\
\hline $\begin{array}{l}\text { Recolección de } \\
\text { leña }\end{array}$ & 37,2 & 42,1 & $3,9^{*}$ & 34,9 & 55,6 & 0,7 \\
\hline $\begin{array}{l}\text { Inv. forestal o } \\
\text { ecoturismo }\end{array}$ & 34,9 & 18,4 & $6,7^{*}$ & 31,7 & 11,1 & 0,9 \\
\hline Reforestación & 39,5 & 7,9 & $11,4^{2}$ & 30,2 & 5,6 & $4,1^{*}$ \\
\hline $\begin{array}{l}\text { Aprovechamiento } \\
\text { maderable }\end{array}$ & 39,5 & 0 & $10,6^{*}$ & 22,2 & 16,7 & 0,1 \\
\hline Cacería & 34,9 & 0 & $8,3^{*}$ & 14,3 & 33,3 & 1,8 \\
\hline $\begin{array}{l}\text { Recolección de } \\
\text { medicinales }\end{array}$ & 16,3 & 21,1 & 3,1 & 12,7 & 0 & 0 \\
\hline $\begin{array}{l}\text { Recolección de } \\
\text { comestibles y } \\
\text { artesanales }\end{array}$ & 4,7 & 18,4 & 0,1 & 15,9 & 27,8 & 1,1 \\
\hline Apicultura & 16,3 & 2,6 & $6,7^{*}$ & 9,5 & 16,7 & 0,1 \\
\hline $\begin{array}{l}\text { Recolección de } \\
\text { forraje }\end{array}$ & 7 & 5,3 & 3,9 & 4,8 & 11,1 & 0,2 \\
\hline
\end{tabular}

Fuente: elaboración propia

$* \mathrm{p}<0,05$

Respecto a la posición al interior de las UD, la recolección de leña $(\mathrm{p}=0,003 \mathrm{I})$ y el cultivo de la milpa $(\mathrm{p}=0,003 \mathrm{I})$ son labores realizadas con mayor probabilidad por hombres y mujeres jefes de hogar. La reforestación es la única actividad influida por la correlación de las tres variables analizadas $(\mathrm{p}=0,008)$, ya que estadísticamente es mayor la probabilidad de que sea realizada por hombres jefes de hogar ejidatarios. Esto se debe a que la reforestación es un requisito de acceso a programas gubernamentales dirigidos principalmente a los ejidatarios, pese a la percepción generalizada sobre la ineficiencia de esta labor basada en que "el monte crece sin ayuda" (Ejidatario, comunicación personal, 24 de mayo de 20I6).

Las UD del ejido utilizan de forma diversa la selva para la subsistencia de sus miembros y en general bajo la coordinación de los hombres jefes de hogar, que realizan la mayoría de actividades y determinan la distribución del terreno, los usos y sus beneficios como parte de una estrategia campesina tradicional o de subsistencia (Gurri, 2010). Aun cuando esta estrategia es compartida por UD ejidatarias y avecindadas en el uso de la selva, salvo ciertas diferencias de acuerdo con la variable de género, al analizar las condiciones de acceso a los distintos tipos de vegetación, tanto el género como la condición de tenencia evidenciaron diferencias. Existe una predilección por parte de los avecindados por el monte alto, que corresponde a la selva mediana subperennifolia y subcaducifolia (Granados et al., 1999), para el uso preferencial del cultivo de sus milpas ( $\mathrm{p}=0$,0I2). Por el contrario, los ejidatarios 
han restringido la rotación de sus cultivos solo al huamil o a vegetación secundaria, como parte de un acuerdo ejidal anterior al proyecto piloto.

Asimismo, se estableció que entre los terrenos utilizados por las UD avecindadas para sus milpas, recolección de leña, especies medicinales y cacería, se encuentran áreas de conservación. Por el contrario, la mayoría de miembros de las UD ejidatarias solo reforestan y participan en actividades de ecoturismo dentro de estas áreas, y establecen las otras actividades en sus parcelas. Esta situación se relaciona con la distribución de la propiedad y las condiciones de accesibilidad que benefician en mayor medida a las UD ejidatarias:

Son $3 \mathrm{~km}$ a la redonda [del centro urbano] donde según la gente va a leñar, pero eso ya está repartido entre los ejidatarios y no puedes tu [avecindado] acceder a esos terrenos, y lo único es que vas a comprar la leña o vas a leñar más lejos. (Representante de una ONG, comunicación personal, 25 de mayo de 2016)

Respecto a las diferencias de acceso a la selva, de acuerdo con el género, se estableció una división espacial entre hombres y mujeres. El monte alto es de dominio exclusivo de los hombres $(\mathrm{p}=0,03)$, mientras que las mujeres realizan sus actividades en el monte o la selva bajos, en la vegetación secundaria y en las áreas de milpa.

\section{Los dispositivos de la territorialización de REDD+}

La consolidación del proyecto piloto en la reserva MKK está ligada a la implementación previa del plan de manejo forestal ejidal de 1986 y del programa de ordenamiento ecológico territorial de 2005. El primero fue parte de un proyecto gubernamentalmente enmarcado en un nuevo esquema de gobernanza forestal comunitario y de avance del paradigma de manejo forestal sustentable (Antinori y Bray, 2005). El ordenamiento territorial con orientación ecológica es impulsado a nivel nacional por la Conafor como el principal instrumento normativo de gobernanza ambiental (García-Frapolli, 2015). En el ejido FCP, el ordenamiento derivó en la organización de cuatros zonas: agrícola, de expansión urbana, forestal y de conservación (U’yo’olché A. C., 2005).

Estos dos dispositivos pueden entenderse como pilares del proyecto piloto, porque generaron capacidades de organización para el manejo forestal entre las UD ejidatarias, integraron la conservación en la planeación comunitaria y condujeron a una mayor capacidad de gestión y posicionamiento del ejido ante agentes extraterritoriales. Dos años después del ordenamiento territorial, se creó la reserva MKK, en una zona previamente determinada de vocación agrícola y en donde aún habitan o trabajan algunas UD ejidatarias y avecindadas.

La reserva MKK es reconocida desde 2010 por la Comisión Nacional de Áreas Naturales Protegidas (Conanp) como la primer Área Destinada Voluntariamente a la Conservación de toda la península de Yucatán (GarcíaFrapolli, 2015). En sus diez años de existencia, ha sido cogestionada por los ejidatarios y la ONG U’yơolché A. C., con asesoría de TNC, Amigos de Sian Ka’an, la Conafor, el PNUD y centros de investigación.

Si bien MKK puede ser considerada como un dispositivo vinculado directamente a REDD+, es con el monitoreo comunitario de carbono que empieza a tomar forma el proyecto piloto. Esta iniciativa ha tenido dos fases: la primera, en $\mathbf{2 0 0 7}$, a partir de un muestreo del proyecto de servicios ambientales para construir un inventario forestal y de $\mathrm{CO}_{2}$ de la selva, requisito para emprender el proceso de certificación para la venta de bonos de carbono, y la segunda fase, de 2012 a 20I6, surge con la iniciativa nacional "Fortalecimiento REDD+ y cooperación Sur-Sur", que tenía como uno de sus componentes el establecimiento de sitios de monitoreo intensivo de carbono (SánchezSantos et al., 2015). El sitio de monitoreo en MKK cubrió zonas de vegetación secundaria y de selva mediana subperennifolia.

El monitoreo comunitario en sus dos fases fue realizado por miembros de UD ejidatarias -en la última participaron mujeres- capacitados y dirigidos por técnicos de U'yo’olché A. C. y del PNUD. Pese a que esta iniciativa no ha sido suficiente para que el ejido acceda a la certificación de bonos de carbono, que se espera puedan 
comercializarse con el sector hotelero de la Riviera Maya, a nivel local contribuyó con la creación de grupos de monitoreo comunitarios de biodiversidad con el apoyo del proyecto Gobernanza Local para REDD+. A nivel nacional, aportó a la construcción del Sistema de Monitoreo, Reporte y Verificación, que ha sido integrado por el Gobierno mexicano a la Iniciativa de Reducción de Emisiones ante el Banco Mundial (Conafor, 2016).

Otro dispositivo identificado son los programas de pago por servicios ambientales (PSA) administrados por la Conafor, que han suplido la ausencia de venta de bonos de carbono para la financiación del funcionamiento de la reserva MMK y de las iniciativas ejidales de conservación y reforestación. Entre 2008 y 2013 se recibieron USD 21.000 anuales por PSA hidrológico, y entre 2011 y 2015, cerca de USD 300.000 del PSA de conservación de la biodiversidad (García-Frapolli, 2015). A estos programas, se suma la creación del centro ecoturístico y de investigación Síijil Noh Ha dentro de la reserva MKK. Este espacio se ha convertido en un sitio de demostración de las iniciativas nacionales de captura de carbono.

Adicionalmente, se identificaron dispositivos que no son reconocidos como parte de REDD+ pero que contribuyen a la adopción de prácticas-modelo sostenibles (Ulloa, 20I3). Algunas de las más relevantes son la implementación de estufas ahorradoras de leña Túumben K’óoben, desarrolladas por U’yo’olché A. C., y los viveros de reforestación como parte del plan de aprovechamiento y zonificación de especies comerciales.

Finalmente, encontramos la disposición de normas de uso y acceso a la selva por parte de autoridades y la asamblea ejidal, formuladas por acuerdos internos y requerimientos extraterritoriales de la Conafor y varias ONG, en muchos casos ligadas al proyecto piloto REDD+. Entre las normas o disposiciones locales se encuentran la prohibición de actividades económicas primarias en las reservas (García-Frapolli, 2015), la regulación de la cacería, el control del transporte de leña, restricciones al sistema roza, tumba y quema (RTQ) en las milpas, señalización en los caminos de terracería y la disposición diferencial de sanciones a ejidatarios y avecindados por infracciones al uso de bienes comunes.

\section{Los intermediarios locales y la interacción local- global}

Identificamos cuatro grupos de agentes e instituciones que inciden, promueven o se ven afectados por la implementación de los dispositivos anteriormente descritos: agentes extraterritoriales, promotores locales, ejidatarios y avecindados. En este caso, nos centramos en el papel de los intermediarios, U'yo’olché A. C., y en los promotores ejidatarios, autoridades ejidales y ejidatarias con capacidad de gestión, por su papel en la traducción y materialización de algunos de los elementos discursivos de REDD+ a partir del proyecto piloto.

Los agentes intermediarios locales, tanto las ONG como los ejidatarios, sirven como mediadores y traductores en los procesos globales y locales ligados a REDD+; interactúan con los agentes extraterritoriales que han promovido la iniciativa, como TNC, la Conafor, el PNUD y Amigos de Sian Ka'an; se actualizan constantemente sobre los avances políticos de REDD + y los enfoques de manejo forestal, y son parte de plataformas regionales de impulso, como el Comité Técnico Consultivo (CTC-REDD+) de Quintana Roo. Asimismo, interactúan con y les traducen los proyectos y las iniciativas forestales y de conservación a las UD ejidatarias organizadas en la asamblea ejidal y a las avecindadas. Por tal razón, la interpretación y reproducción discursiva de REDD+ por parte de este grupo de agentes incide en la forma como se territorializa el proyecto piloto en MKK (tabla 3, 3a). En este caso, indagamos en sus declaraciones sus concepciones sobre la selva y la comunidad -en tanto son los principales objetos de intervención de REDD+- y el establecimiento de posiciones subjetivas, juicios de valor y prácticas modelo que hacen parte de la categoría de construcción del problema. 
TABLA 3

SínTESIS DE REPRESENTACIONES DE LA SELVA Y LA COMUNIDAD POR LOS AGENTES LOCALES

\begin{tabular}{|c|c|c|c|}
\hline & Dimensión & ONG local & Promotores ejidatarios \\
\hline \multirow{3}{*}{$\frac{\sum^{\circ}}{\mathscr{D}}$} & ¿Cómo está? & $\begin{array}{l}\text { Selva en regeneración con vegetación secundaria y } \\
\text { parches de ganadería y agricultura }\end{array}$ & $\begin{array}{l}\text { Monte alto y bajo en buen estado, intercalado con } \\
\text { zonas de huamil y milpas }\end{array}$ \\
\hline & Usos posibles & $\begin{array}{ll}\text { - Extracción de madera } & \\
\text { - Pago por servicios } & \text { - Ecoturismo } \\
\text { ambientales } & \text { - Protección } \\
\text { - Agricultura } & \text { - Investigación } \\
\text { sostenible } & \end{array}$ & $\begin{array}{l}\text { - Extracción de } \\
\text { madera } \\
\text { - Pago por servicios } \\
\text { ambientales } \\
\text { - Milpa (sin quema) }\end{array}$ \\
\hline & $\begin{array}{l}\text { Premisas } \\
\text { asociadas a la } \\
\text { selva }\end{array}$ & $\begin{array}{l}\text { - Biodiversidad } \\
\text { - Tradición } \\
\text { - Bienestar }\end{array}$ & $\begin{array}{l}\text { - Conservación } \\
\text { - Identidad }\end{array}$ \\
\hline \multirow{4}{*}{ 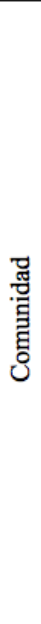 } & Clasificación & $\begin{array}{l}\text { - Ejido (institución) } \\
\text { - Ejidatarios } \\
\text { - Sectores vulnerables: mujeres, jóvenes y } \\
\text { avecindados }\end{array}$ & $\begin{array}{l}\text { - Quienes conservan: ejidatarios y sus UD } \\
\text { - Quienes destruyen: UD avecindadas y algunos } \\
\text { ejidatarios }\end{array}$ \\
\hline & $\begin{array}{l}\text { Contexto } \\
\text { comunitario }\end{array}$ & $\begin{array}{l}\text { - Comunidad dependiente de la selva, con prácticas } \\
\text { cada vez más sostenibles } \\
\text { - Desconfianza y conflictos internos por proyectos } \\
\text { - Resistencia de ejidatarios a la participación de } \\
\text { mujeres y jóvenes en los proyectos } \\
\text { - Poca incidencia de los avecindados }\end{array}$ & $\begin{array}{l}\text { - Todos dependen de la selva para su } \\
\text { subsistencia, aunque los jóvenes están migrando } \\
\text { a las ciudades } \\
\text { - Conflicto con algunos ejidatarios y avecindados } \\
\text { que no respetan los acuerdos ejidales y siguen } \\
\text { degradando la selva }\end{array}$ \\
\hline & $\begin{array}{l}\text { ¿Quiénes se } \\
\text { pueden } \\
\text { beneficiar de } \\
\text { REDD+? }\end{array}$ & $\begin{array}{l}\text { - Propietarios-ejidatarios } \\
\text { - Usuarios con permiso del ejido (avecindados, } \\
\text { mujeres y jóvenes) }\end{array}$ & $\begin{array}{l}\text { - Ejidatarios con reconocimiento comunitario o } \\
\text { de la selva y mujeres y jóvenes de UD } \\
\text { ejidatarias }\end{array}$ \\
\hline & $\begin{array}{l}\text { ¿Qué deben } \\
\text { hacer? }\end{array}$ & $\begin{array}{l}\text { - Implementar prácticas sostenibles } \\
\text { - Adoptar las reglas de uso y acceso a la selva } \\
\text { - Participar en actividades de conservación }\end{array}$ & $\begin{array}{l}\text { - Adoptar las reglas de uso y acceso a la selva } \\
\text { - Denunciar acciones que perturben la selva } \\
\text { - Participar en la organización ejidal } \\
\text { - Participar en actividades de conservación }\end{array}$ \\
\hline
\end{tabular}

Fuente: elaboración propia

\section{Discusión}

El caso del ejido FCP resalta la existencia de múltiples agentes e intereses que tienen un papel central en la traducción de políticas a nivel local. En este caso particular, los intermediarios locales traducen y reproducen el campo discursivo de REDD+ a partir de lo que Pasgaard (2015) denominó como una dinámica bidireccional de retroalimentación y un conflicto entre lo local, los espacios regionales de formulación y las negociaciones internacionales. Vale señalar que nos referimos a campo discursivo ya que planteamos que en la estrategia REDD+, de acuerdo con la dinámica nacional y local, se integran, movilizan e incluso contraponen discursos hegemónicos de gobernanza (Holmgren, 2013), conservación, desarrollo (Büscher y Dressler, 2007) y mercantilización de la naturaleza (Pistorius et al., 20I2), entre otros. 
Esta dinámica bidireccional se evidencia, por ejemplo, en la adopción por parte de los promotores ejidatarios de una retórica conservacionista (Frazier, 2006), que conlleva a una transformación en la concepción de la selva como fuente de subsistencia hacia una reivindicación como "pulmón del ejido", lo que resalta una apropiación local de la concepción de los bosques como sumideros de carbono (Gupta et al., 20I2). Este proceso, si bien es discursivo, está ligado a las disposiciones normativas que restringen el uso productivo de la selva y favorecen la vocación comunitaria de prestación de servicios a través de la conservación, la reforestación y el turismo (Pasgaard, 2015).

Asimismo, esta postura conservacionista ha llevado al fortalecimiento de la división comunitaria entre quienes son propietarios de la selva y quienes son únicamente usuarios. La lógica de protección frente a la degradación ambiental refuerza los mecanismos tradicionales de distribución de beneficios y participación en proyectos, ligados al tipo de tenencia de la tierra, y fortalece relaciones socioespaciales de diferenciación y exclusión entre algunos agentes y sectores del ejido que son reforzadas a su vez por agentes y dispositivos que están localizados en espacios extraterritoriales. Este es el caso, por ejemplo, del marco normativo gubernamental que establece como beneficiarios directos a los propietarios del carbono de los bosques (Conafor, 2016). Los ejidatarios, así como las UD avecindadas y, en menor grado, las mujeres y jóvenes de UD ejidatarias, son excluidos por su condición de no propietarios de la tierra.

Estos procesos de traducción y materialización en dispositivos por parte de los intermediarios no necesariamente se llevan a cabo en consenso, como se evidenció con las posiciones encontradas respecto a la sustitución (Conafor, 2016) o la reivindicación cultural de los ejidatarios hacia el sistema de cultivo RTQ, como expresión local de un amplio debate sobre su impacto en las emisiones de carbono (Bruun, Neergaard, Lawrence y Ziegler, 2009). Esta situación permite ver la existencia de múltiples intereses que definen la forma en que se traducen y reproducen los discursos, que en el caso de FCP se sustentan en la territorialidad de cada grupo de agentes, las expectativas con el proyecto piloto, las relaciones comunitarias, el acceso y uso de la selva y las relaciones de poder a nivel local.

\section{Efectos de la territorialización de REDD+}

Los dispositivos vinculados al proyecto piloto REDD+ como mecanismos discursivos utilizados por los intermediarios locales y agentes extraterritoriales generan efectos especialmente en las dinámicas de uso y acceso a la selva (figura 2). A su vez, tales efectos, junto con otros factores del contexto sociohistórico ejidal, como la tenencia de la tierra, la organización comunitaria y la vocación y distribución del espacio, determinan la forma como las UD ejidatarias y avecindadas de FCP interpretan y se posicionan sobre los dispositivos del proyecto.

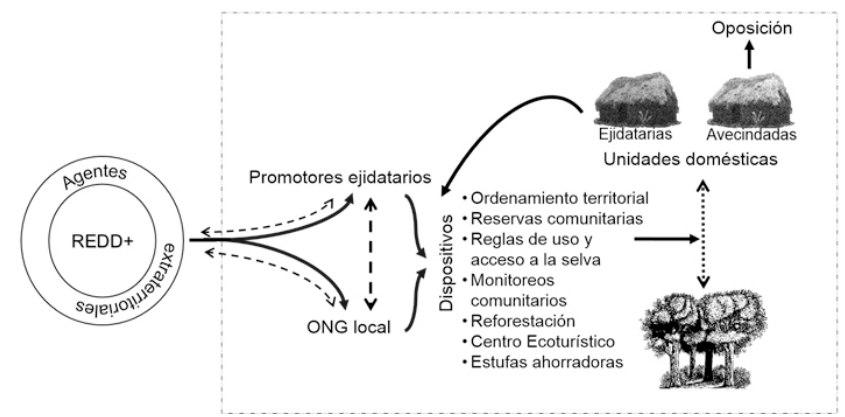

FIGURA 2.

Territorialización de REDD+ En El EJido FCP

Nota: las flechas solidas representan el proceso de traducción del campo discursivo de la iniciativa; las flechas entrecortadas simbolizan la interacción entre los agentes, y la línea punteada, la relación selva-unidades domésticas.

Fuente: elaboración propia con base en Referencias visuales tomadas de Pixabay.com

Los efectos de los dispositivos que preceden o hacen parte del piloto REDD+ se pueden categorizar en tres tipos:

I) integración, 2) restricción-conflicto y 3) diversificación-transformación. Cabe mencionar que a pesar de que las mujeres entrevistadas no reconocieron efectos particulares en sus interacciones con la selva -posiblemente por las 
relaciones domésticas de poder que limitan el acceso físico y generan un extrañamiento de las mujeres hacia los bosques (Rocheleau y Edmunds, 1997)-, sí existen algunos efectos de la territorialización de REDD+ que afectan de forma diferencial a hombres y mujeres.

Dentro de las dinámicas de integración resaltamos la adopción, por parte de promotores locales y gran parte de los ejidatarios, del discurso hegemónico de conservación y de manejo comunitario del territorio, que se ha expandido por la península de Yucatán (Frazier, 2006). La adopción de este discurso es producto de y a su vez facilita la inserción comunitaria en plataformas de consulta y diseño de políticas ambientales y la creación de alianzas con ONG interesadas en la conservación y el manejo sustentable forestal en Quintana Roo.

Asimismo, la presión ejercida por los agentes extraterritoriales para integrar enfoques de género y de participación comunitaria, sumada al cambio generacional y las brechas educativas, ha propiciado la participación de mujeres y jóvenes de UD ejidatarias en proyectos e iniciativas comunitarios. Sin embargo, la inserción se da únicamente en la etapa de implementación, condición que, según investigaciones en distintos proyectos de REDD +, limita la participación comunitaria por la no inclusión de sus intereses y conocimientos (Larson et al., 2015).

Por otro lado, la implementación y el reforzamiento de dispositivos normativos sobre el uso y acceso a la selva afectan diferencialmente a las UD ejidatarias y avecindadas. Mientras los ejidatarios asentados en la reserva MKK pueden acceder a permisos de aprovechamiento sostenible de sus terrenos, las UD avecindadas están sujetas a restricciones y sanciones. Destacamos la prohibición de RTQ en áreas de vegetación madura y secundaria, que podría tener el efecto contrario de afectar la productividad de los cultivos y la propia regeneración de la selva (Borrego, Salinas-Melgoza, Skutsch, 2015). Resaltamos también la regulación de la recolección de leña -actividad realizada por las mujeres- a través de permisos especiales y cuotas de extracción, la prohibición de la cacería de subsistencia dentro de la reserva y la restricción de movilidad en áreas del monitoreo comunitario de carbono.

Estas restricciones afectan particularmente a quienes no poseen propiedad de la tierra (Poudyal et al., 2016), en este caso las UD avecindadas que se encuentran asentadas dentro de la reserva MKK. Además, este sector, en el contexto ejidal mexicano, tiene poca incidencia en las decisiones ejidales y reducido acceso a compensaciones o participación en los proyectos (Navarro-Olmedo, Haenn, Schmook y Radel, 2015). Esta situación ha derivado en el surgimiento y escalamiento de un conflicto entre algunas UD avecindadas y los intermediarios locales, a raíz de las demandas de permanencia en la zona y de una nueva delimitación de la reserva que reconozca los asentamientos previos.

La otra cara de los dispositivos desplegados en el ejido ha sido el impulso de acciones de reforestación y aprovechamiento forestal, así como la posibilidad de acceso a mecanismos financieros de conservación y prestación de servicios ambientales (García-Frapolli 20I5). Estas actividades han aportado a la diversificación y transformación de las fuentes de trabajo e ingresos de algunas UD ejidatarias, como parte de un proceso de reconversión de economías primarias a terciarias o de prestación de servicios, que ha sido impulsado por estrategias neoliberales de conservación (Durand, 20I4), como el propio REDD+.

Sin embargo, esta reconversión podría llevar a una mayor dependencia de fuentes externas de ingreso y a una inseguridad alimentaria (Ibarra et al., 20II). Adicionalmente, hay una resignificación del interés por la conservación ligada al mercado, a la gestión ejidal del territorio y a tener mayor visibilidad institucional, que garantiza el apoyo técnico y financiero extraterritorial a los proyectos del ejido.

\section{Conclusiones}

El complejo proceso de territorialización de la estrategia global de REDD+ dentro del ejido Felipe Carrillo Puerto integra múltiples actores y relaciones de traducción discursiva desde el nivel global hasta el local. En 
esta traducción intervienen agentes extraterritoriales, como ONG internacionales, entidades gubernamentales y multilaterales, agentes locales que fungen como intermediarios y las unidades domésticas con y sin derecho a la tenencia de la tierra.

Este conjunto de agentes e instituciones tejen una red heterogénea de intereses mediados por múltiples territorialidades y concepciones sobre cómo las comunidades deben relacionarse y aprovechar los bosques. Esta heterogeneidad resalta la existencia de múltiples relaciones que generan o refuerzan diferencias socioespaciales, entre las que se resaltan asimetrías de poder entre los agentes a nivel comunitario y doméstico.

La territorialización del proyecto piloto REDD+ en el ejido genera efectos que complejizan las dinámicas internas y externas de las unidades domésticas y su relación con la selva y el territorio. La articulación del campo discursivo y los dispositivos de REDD+ con las prácticas materiales y simbólicas del ejido promueve cambios en las estrategias de subsistencia de las unidades domésticas. La focalización hacia el sector ejidatario y la inserción de agentes extraterritoriales en el territorio contribuyen a un escenario contrastante de apertura a la participación de mujeres y jóvenes en la gestión del territorio, pero también de mayor exclusión de las unidades domesticas avecindadas.

Por otro lado, los vínculos institucionales de REDD+ con un discurso hegemónico de conservación neoliberal, mediado por las fuerzas de mercado, introducen una nueva valoración de la selva por su condición de sumidero de carbono. Ello lleva a la adopción de medidas que modifican las relaciones entre las unidades domésticas y la selva hacia un ámbito de sustentabilidad de estas como prestadoras de servicios ambientales, y riñe con algunas prácticas productivas y domésticas consideradas como centrales para la subsistencia en economías campesinas.

Si bien la territorialización de REDD+ ha favorecido la integración y diversificación de valoraciones, fuentes de ingresos e intereses ligados a la conservación, aún existe la necesidad y oportunidad de fomentar un proceso que integre de mejor manera los distintos agentes de la comunidad, sus relaciones y sus valoraciones sobre la selva. Por tanto, compartimos dos posibles rutas de reflexión y acción ante los retos de gobernanza, sostenibilidad y bienestar que se le han planteado a REDD+ como actual -y transitorio (Lund, Sungusia, Bukhi-Mabele y Scheba, 2017)marco global de gobernanza forestal.

Una primera ruta, desarrollada desde el ámbito institucional, es el reforzamiento de las salvaguardas o garantías de transparencia con respeto a derechos indígenas y comunitarios, participación y conservación, entre otros. Esta ruta se evidencia en investigaciones como la de Shepherd y Ludlow-Paz (2015), sobre la creación de mecanismos de participación más incluyentes que los ejidos, y la de Rodríguez-Canto et al. (2016), sobre la milpa de roza-tumbaquema.

La segunda ruta parte de experiencias comunitarias y organizaciones sociales y civiles que han optado por reformar (Aguilar-Støen, 2017) o crear iniciativas REDD+ desde las realidades territoriales. Tal es el caso de la alternativa indígena a REDD+ de la Coordinadora de las Organizaciones Indígenas de la Cuenca Amazónica o bien de las propuestas comunitarias como Reddeldia, en Chiapas, México, y el pueblo indígena bribri, en Costa Rica, que optan por la oposición a REDD+y apuestan por iniciativas locales de manejo comunitario de los bosques para la subsistencia y para disminuir localmente sus emisiones de $\mathrm{CO}_{2}$.

\section{Agradecimientos}

El Dr. Gustavo Blanco agradece el apoyo de los proyectos Fondecyt 1160857 y Fondap I5II0009 de la Comisión Nacional de Investigación Científica y Tecnológica de Chile (Conicyt). 


\section{Referencias}

Agrawal, A., y Gibson, C. (1999). Enchantment and disenchantment: The role of community in natural resource conservation. World Development, 27(4), 629-649. https://doi.org/10.10I6/S0305-750X(98)00I6I-2

Aguilar-Støen, M. (2017). Better safe than sorry? Indigenous peoples, carbon cowboys and the governance of REDD in the Amazon. Forum for Development Studies, 44(I), 91-108. https://doi.org/I0.1080/08039410.2016.1276098

Anderson, B., y McFarlane, C. (20II). Assemblage and geography. Area, 43(2), I24-I27. https://doi.org/10.IIII/ j.1475-4762.2011.01004.X

Antinori, C., y Bray, D. (2005). Community forest enterprises as entrepreneurial firms: Economic and institutional perspectives from Mexico. World Development, 33, 1529-1543. https://doi.org/10.1016/j.worlddev.2004.10.0II

Awono, A., Somorin, O., Eba’a Atyi, R., y Levang, P. (20I4). Tenure and participation in local REDD+ projects: Insights from southern Cameroon. Environmental Science and Policy, 35, 76-86. https://doi.org/10.1016/j. envsci.2013.01.017

Benjaminsen, G. (2017). The bricolage of REDD+ in Zanzibar: From global environmental policy framework to community forest management. Journal of Eastern African Studies, II(3), 506-525. https://doi.org/I0.I080/ 17531055.2017.1357103

Blanco, G., Arce, A., y Fisher, E. (2015). Becoming a region, becoming global, becoming imperceptible: Territorialising salmon in chilean Patagonia. Journal of Rural Studies, 42, I79-190. https://doi.org/I0.I0I6/ j.jrurstud.2015.10.007

Blanco, G., y Fuenzalida, M. (2013). La construcción de agendas científicas sobre cambio climático y su influencia en la territorialización de políticas públicas: reflexiones a partir del caso chileno. En J. Postigo (ed.), Cambio climático, movimientos sociales y políticas públicas. Una vinculación necesaria (pp. 77-104). Santiago: Clacso, ICAL.

Borrego, A., Salinas-Melgoza, M., y Skutsch, M. (2015). El valor de las emisiones de carbono asociadas al sistema agrícola de roza, tumba y quema. En F. Paz Pellat, J. Wong González y R. Torres Alamilla (eds.), Estado actual del conocimiento del ciclo del carbono y sus interacciones en México: síntesis a 2015 (pp. 578-586). Villahermosa, México.

Bray, D., Merino, L., y Barry, D. (eds). (2007). Los bosques comunitarios de México. Manejo sustentable de paisajes forestales. México: Instituto Nacional de Ecología.

Bruun, T., Neergaard, A. de, Lawrence, D., y Ziegler, A. (2009). Environmental consequences of the demise in swidden agriculture in Southeast Asia: Carbon storage and soil quality. Human Ecology, 37, 375-388. https ://doi.org/10.1007/s10745-009-9258-x

Büscher, B., y Dressler, W. (2007) Linking neoprotectionism and environmental gobernance: On the rapidly increasing tensions between actors in the environment-development nexus. Conservation and Society, ${ }_{5}(4)$, 586-6II. Recuperado de https://repository.up.ac.za/bitstream/handle/2263/5624/B\%C3\%BCscher_Linking $\% 282007 \% 29$.pdf?sequence $=\mathrm{I}$

Comisión Nacional Forestal [Conafor]. (2016). Documento de la Iniciativa de Reducción de Emisiones (IRE). Jalisco: autor.

Comisión Nacional Forestal [Conafor]. (2017). Estrategia Nacional para REDD+ (ENAREDD+). Jalisco: autor.

Congreso de los Estados Unidos Mexicanos. (1992). Ley Agraria. Recuperado de https://www.juridicas.unam.m $\mathrm{x} /$ legislacion/ordenamiento/ley-agraria\#II3I8 
Corbera, E., y Schroeder, H. (20II). Governing and implementing REDD+. Environmental Science \& Policy, I4(2), 89-99. https://doi.org/10.1016/j.envsci.2010.II.002

Cronkleton, P., Bray, D., y Medina, G. (20II). Community forest management and the emergence of multi-scale governance institutions: Lessons for REDD+ development from Mexico, Brazil and Bolivia. Forests, 2(2), 45I-473. https://doi.org/10.3390/f202045I

DeLanda, M. (2006). A new philosophy of society: Assemblage theory and social complexity. Continuum, 40(5), I42-I42. https://doi.org/I0.II77/0263276407086408

Durand, L. (20I4). ¿Todos ganan? Neoliberalismo, naturaleza y conservación en México. Sociológica, 29(82), 183-223. Recuperado de http://www.scielo.org.mx/pdf/soc/v29n82/v29n82a6.pdf

Ellis, E., y Porter-Bolland, L. (2008). Is community-based forest management more effective than protected areas? A comparison of land use/land cover change in two neighboring study areas of the Central Yucatan Peninsula, Mexico. Forest Ecology and Management, 256(II), 197I-I983. https://doi.org/I0.I0I6/j.foreco.200 8.07 .036

Frazier, J. (2006). Biosphere reserves and the "Yucatan" syndrome: Another look at the role of NGOs. Landscape and Urban Planning, 74(3-4), 313-333. https://doi.org/10.1016/j.landurbplan.2004.09.010

Gallemore, C., Gregorio, M. di, Brockhaus, M., Fatorelli, L., y Muharrom, E. (2017). How institutions and beliefs affect environmental discourse: Evidence from an eight-country survey on REDD+. Global Environmental Change, 45, 133-150. https://doi.org/10.1016/j.gloenvcha.2017.05.006

García-Frapolli, E. (2015). Con y sin el mercado. Diversificación de iniciativas de conservación comunitaria en el ejido de Felipe Carrillo Puerto, México. Otra Economía, 9(17), 151-163. Recuperado de http://revistas.unisi nos.br/index.php/otraeconomia/article/view/otra.2015.917.04

Granados, D., Florencia, G., Ríos, L., y Murcia, E. (1999). La milpa en la zona maya de Quintana Roo. Revista de Geografía Agrícola, I-72. Recuperado de https://chapingo.mx/revistas/revistas/articulos/doc/rga-1536.pdf

Gupta, A., Lövbrand, E., Turnhout, E., y Vijge, M. (20I2). In pursuit of carbon accountability: The politics of REDD+ measuring, reporting and verification systems. Current Opinion in Environmental Sustainability, 4(6), 726-731. https://doi.org/10.1016/j.cosust.2012.10.004

Gurri, F. (2010). Smallholder land use in the southern Yucatan: How culture and history matter. Regional Environmental Change, IO(3), 219-231. https://doi.org/I0.1007/sıoII3-010-0II4-8

Harris, O. (1986). La unidad doméstica como una unidad natural. Nueva Antropología, 8(30), 199-222. Recuperado de http://www.redalyc.org/pdf/159/15903010.pdf

Holmgren, S. (2013). REDD+ in the making: Orders of knowledge in the climate-deforestation nexus. Environmental Science and Policy, 33, 369-377. https://doi.org/10.1016/j.envsci.2013.04.007

Ibarra, J., Barreau, A., del Campo, C., Camacho, C., Martin, G., y McCandless, S. (20rI). When formal and marketbased conservation mechanism disrupt food sovereignty: Impacts of community conservation ad payments for environmental services on an indigenous community of Oaxaca, Mexico. International Forestry Review, 13(3), 318-337. https://doi.org/10.1505/146554811798293935

Intergovernmental Panel on Climate Change [IPCC]. (2014). Climate Change 20I4: Synthesis Report. Contribution of Working Groups I, II and III to the Fifth Assessment Report of the Intergovernmental Panel on Climate Change. En R. K. Pachauri y L. A. Meyer (eds.). Geneva: autor.

Keller, R. (20II). The sociology of knowledge approach to discourse (SKAD). Human Studies, 34(I), 43-65. https ://doi.org/10.1007/s10746-0II-9175-Z 
Keller, R. (2012). Entering discourses: A new agenda for qualitative research and sociology of knowledge. Qualitative Sociology Review, 8(2), 46-75. Recuperado de http://www.qualitativesociologyreview.org/ENG Nolume22/QSR_8_2_Keller.pdf

Larson, A. (20II). Forest tenure reform in the age of climate change: Lessons for REDD+. Global Environmental Change, 2I(2), 540-549. https://doi.org/10.10I6/j.gloenvcha.2010.II.008

Larson, A., Dokken, T., Duchelle, A., Atmadja, S., Resosudarmo, I., Cronkleton, P., ... Selaya, G. (2015). The role of women in early REDD+ implementation: Lessons for future engagement. International Forestry Review, I7(I), 43-65. https://doi.org/10.1505/I46554815814725031

Lund, J., Sungusia, E., Bukhi-Mabele, M., y Scheba, A. (2017). Promising change, delivering continuity: REDD+ as conservation fad. World Development, 89, I24-I39. https://doi.org/ı0.10I6/j.worlddev.2016.08.005

McNetting, R., Wilk, R., y Arnould, E. (1985). Households: Comparative and historical studies of the domestic group. American Ethnologist, I2(3). https://doi.org/I0.1525/ae.1985.12.3.02a00210

Méndez-López, M., García-Frapolli, E., Ruiz-Mallén, I., Porter-Bolland, L., y Reyes-Garcia, V. (2015). From paper to forest: Local motives for participation in different conservation initiatives. Case studies in southeastern Mexico. Environmental Management, 56(3), 695-708. https://doi.org/10.1007/s00267-015-0522-0

Merino, L., Rodríguez, J., Ortíz, G., y García, A. (2008). Estudio estratégico sobre el sector forestal mexicano. México: Consejo Civil Mexicano para la Silvicultura Sostenible A. C.

Morett-Sánchez, J., y Cosío, C. (2017). Panorama de los ejidos y comunidades agrarias en México. Agricultura, Sociedad y Desarrollo, I4(I), I25-I52. Recuperado de http://www.scielo.org.mx/pdf/asd/vi4nI/I870-5472-asd -I4-0I-00I25-en.pdf

Moure-Peña, M. (2013). Desarrollo sustentable ante el cambio climático: ¿qué papel puede tener REDD+ en las comunidades locales? México: El Colegio de la Frontera Sur.

Navarro-Olmedo, S., Haenn, N., Schmook, B., y Radel, C. (2015). The legacy of Mexico's agrarian counterreforms: Reinforcing social hierarchies in Calakmul, Campeche. Journal of Agrarian Change, I6(I), 3-23. h ttps://doi.org/ı0.IIII/joac.I2095

Newton, P., Miller, D., Byenkya, M., y Agrawal, A. (2016). Who are forest-dependent people? A taxonomy to aid livelihood and land use decision-making in forested regions. Land Use Policy, 57, 388-395. https://doi.org/ I0.1016/j.landusepol.2016.05.032

Pasgaard, M. (2015). Lost in translation? How project actors shape REDD+ policy and outcomes in Cambodia. Asia Pacific Viewpoint, 56(I), III-I27. https://doi.org/ I0.IIII/apv.I2082

Pimienta-Lastra, R. (2000). Encuestas probabilísticas vs. no probabilísticas. Política y Cultura, (13), 263-276. Recuperado de http://www.redalyc.org/articulo.oa?id=26701313

Pistorius, T., Schaich, H., Winkel, G., Plieninger, T., Bieling, C., Konold, W., y Vold, K.-R. (2012). Lessons for REDDplus: A comparative analysis of the German discourse on forest functions and the global ecosystem services debate. Forest Policy and Economics, I8, 4-I2. https://doi.org/I0.I016/j.forpol.2011.09.00I

Poudyal, M., Ramamonjisoa, B., Hockley, N., Rakotonarivo, O., Gibbons, J., Mandimbiniaina, R., y Jones, J. (2016). Can REDD+ social safeguards reach the 'right' people? Lessons from Madagascar. Global Environmental Change, 37, 3I-42. https://doi.org/10.1016/j.gloenvcha.2016.01.004

Rocheleau, D., y Edmunds, D. (1997). Women, men and trees: Gender, power and property in forest and agrarian landscapes. World Development, 25(8), 1351-1371. https://doi.org/10.1016/S0305-750X(97)00036-3 
Rodríguez-Canto, A., González, P., Flores, J., Nava, R., Dzib, L., Pérez, J., ... González, A. (2016). Milpas de las comunidades mayas y dinámica de uso del suelo en la Península de Yucatán. México: Universidad Autónoma de Chapingo.

Sánchez-Santos, G., Arreola, J., López-Merlín, D., Maldonado, V., Olguín, A., Wayson, C., ... Puc, R. (2015). Sitio de monitoreo intensivo del carbono en Quintana Roo. México: Secretaría de Medio Ambiente y Recursos Naturales (Semarnat), Estados Unidos Mexicanos, Comisión Nacional Forestal (Conafor), Fortalecimiento REDD+ y cooperación Sur-Sur, Norwegian Ministry of Foreing Affairs, Programa de las Naciones Unidas para el Desarrollo (PNUD), Organización de las Naciones Unidas para la Alimentación y la Agricultura (FAO).

Servicios Ecosistémicos de la Selva Maya [SESM]. (20II). Plan Vivo Project Design Document: Much Kanan K’aax. Quintana Roo: autor.

Shepherd, G., y Ludlow-Paz, L. (2015). Análisis de dependencia forestal y sus implicaciones en la distribución equitativa de beneficios REDD+ en la Península de Yucatán, México. San José: Unión Internacional para la Conservación de la Naturaleza (UICN).

Sunderlin, W., Larson, A., Duchelle, A., Resosudarmo, I., Huynh, T., Awono, A., y Dokken, T. (2014). How are REDD+ proponents addressing tenure problems? Evidence from Brazil, Cameroon, Tanzania, Indonesia, and Vietnam. World Development, 55, 37-52. https://doi.org/10.1016/j.worlddev.2013.01.013

Ulloa, A. (2013). Controlando la naturaleza: ambientalismo transnacional y negociaciones locales en torno al cambio climático en territorios indígenas en Colombia. Iberoamericana, I3(49), I17-133. http://dx.doi.org/I 0.1844I/ibam.13.2013.49.117-133

U'yo’olché A. C. (2005). Estudio de ordenamiento territorial del ejido Felipe Carrillo Puerto. Quintana Roo: Comisión Nacional Forestal (Conafor), TRL, autor.

Wilk, R. (1994). Household ecology: Economic change and domestic life among the Kekchi Maya of Belize. Tucson: University of Arizona Press. https://doi.org/10.1525/jlca.1994.6.1.56.2

Woods, M. (2015). Territorialisation and the assemblage of rural place. En J. Dessein, E. Battaglini y L. Horlings (eds.), Cultural sustainability and region development. Theories and practices of territorialisation. Nueva York: Routledge.

\section{Notas}

*Artículo de investigación

Licencia Creative Commons CC-BY 4.0 